SILVA, N.F.; SONNENBERG, P.E.; BORGES, J.D. Crescimento e produção de cúrcuma (Curcuma longa L.) em função de adubação mineral e densidade de plantio. Horticultura Brasileira, Brasília, v.22, n.1, p.61-65, jan-mar 2004.

\title{
Crescimento e produção de cúrcuma (Curcuma longa L.) em função de adubação mineral e densidade de plantio ${ }^{1}$
}

\author{
Natan Fontoura da Silva; Peter Ernst Sonnenberg; Jácomo Divino Borges \\ UFG, C. Postal 131, 74001-970 Goiânia-GO; E-mail: natan@agro.ufg.br
}

\section{RESUMO}

Avaliou-se o crescimento e a produção de cúrcuma em função de adubação mineral e população de plantas em dois experimentos na Universidade Federal de Goiás, Goiânia (GO). No primeiro, realizado de janeiro a agosto/98, estudaram-se, numa matriz baconiana, doses de uréia, superfosfato triplo e cloreto de potássio $(0 ; 40 ; 80$; $120 ; 160$ e $200 \mathrm{~kg} / \mathrm{ha} \mathrm{de} \mathrm{N} ; 0 ; 50 ; 100 ; 150 ; 250$ e $400 \mathrm{~kg} / \mathrm{ha} \mathrm{de} \mathrm{P}_{2} \mathrm{O}_{5}$ e $0 ; 40 ; 80 ; 120 ; 200 ; 280 \mathrm{~kg} / \mathrm{ha}$ de $\mathrm{K}_{2} \mathrm{O}$, densidades de plantio (55.556; 41.667 e 33.333 plantas/ha, no espaçamento de $0,60 \mathrm{~m}$ entre linhas e $71.428 ; 47.619$ e 35.714 plantas/ha no espaçamento de $0,70 \mathrm{~m}$ entre linhas) e parcelamentos da adubação nitrogenada [toda no plantio, $1 / 2$ no plantio e $1 / 2$ aos três meses após o plantio (AP), 1/ 3 no plantio, $1 / 3$ aos dois meses e $1 / 3$ aos quatro meses AP, $1 / 4$ no plantio, $1 / 4$ a um mês e meio, $1 / 4$ aos 3 meses e $1 / 4$ aos quatro meses e meio AP. No segundo experimento, realizado de dezembro/ 99 a setembro/00, estudaram-se, num fatorial de $2 \times 4$, dois espaçamentos entre linhas $(0,60$ e $0,80 \mathrm{~m})$ e seis espaçamentos entre plantas na linha $(0,05 ; 0,10 ; 0,15 ; 0,20 ; 0,30$ e $0,40 \mathrm{~m})$. O delineamento utilizado foi o de blocos ao acaso com quatro repetições. A produtividade de rizomas frescos aumentou, em função do $\mathrm{N}$, de $12.130 \mathrm{~kg} / \mathrm{ha}$, com a dose zero, até o máximo de $16.124 \mathrm{~kg} / \mathrm{ha}$, com a dose de $130 \mathrm{~kg} / \mathrm{ha}$; e em função do $\mathrm{P}$, de $12.799 \mathrm{~kg} / \mathrm{ha}$, com a dose zero, até o máximo de $15.763 \mathrm{~kg} / \mathrm{ha}$ com a dose de $177 \mathrm{~kg} / \mathrm{ha}$ de $\mathrm{P}_{2} \mathrm{O}_{5}$. O K e o parcelamento de $\mathrm{N}$ não influenciaram a altura das plantas e a produção de rizomas. A produtividade decresceu de $25 \mathrm{t} /$ ha, obtida com o espaçamento de $5 \mathrm{~cm}$ entre plantas, para $18 \mathrm{t} / \mathrm{ha}$ com o espaçamento de $40 \mathrm{~cm}$. Os ganhos de produtividade obtidos em espaçamentos menores que $10 \mathrm{~cm}$ não superaram as diferenças de gastos de rizomas para os respectivos plantios.

Palavras-chave: Curcuma longa, açafrão, população de plantas, adubação, plantas medicinais.

\begin{abstract}
Growth and production of turmeric as a result of mineral fertilizer and planting density

Turmeric growth and production as a result of mineral fertilizer and planting density were evaluated through two experiments carried out at the Universidade Federal de Goiás, Goiânia, Brazil. The first experiment was carried out from January to August 1998. Doses of urea, triple superphosphate and potassium chloride were the treatments $(0 ; 40 ; 80 ; 120 ; 160$ and $200 \mathrm{~kg} /$ ha of $\mathrm{N} ; 0 ; 50 ; 100 ; 150$; 250 and $400 \mathrm{~kg} / \mathrm{ha}$ of $\mathrm{P}_{2} \mathrm{O}_{5}$ and $0 ; 40 ; 80 ; 120 ; 200$ and $280 \mathrm{~kg} / \mathrm{ha}$ of $\left.\mathrm{K}_{2} \mathrm{O}\right)$, planting densities $(55,556 ; 41,667$ and 33,333 plants/ha, spaced $0.60 \mathrm{~m}$ between rows and 71,$428 ; 47,619$ and 35,714 plants/ha spaced $0.70 \mathrm{~m}$ between rows) and $\mathrm{N}$ splitting on side dressing applications [all at planting date (PD); $1 / 2$ at PD and $1 / 2$ three months later; $1 / 3$ at PD, $1 / 3$ two months and $1 / 3$ four months later; $1 / 4$ at PD, 1/4 a month and a half later, $1 / 4$ three months and $1 / 4$ four months and a half later]. The second experiment was carried out from December 1999 to September 2000. and the treatments tested were two row spacings $(0.60$ and $0.80 \mathrm{~m})$ and six within-row spacings $(0.05 ; 0.10$; $0.15 ; 0.20 ; 0.30$ and $0.40 \mathrm{~m})$, displayed in a factorial $2 \times 4$. The experimental design was a complete randomized block with four replicates. The yield of fresh rhizomes increased as a result of $\mathrm{N}$ application from $12,130 \mathrm{~kg} / \mathrm{ha}$ with dose zero, to $16,124 \mathrm{~kg} / \mathrm{ha}$ with the dose of $130 \mathrm{~kg} / \mathrm{ha}$, and as a function of $\mathrm{P}$ application from 12,799 $\mathrm{kg} / \mathrm{ha}$, with dose zero, to $15,763 \mathrm{~kg} / \mathrm{ha}$ with the dose of $177 \mathrm{~kg} / \mathrm{ha}$ of $\mathrm{P}_{2} \mathrm{O}_{5} . \mathrm{K}$ and the splitting of $\mathrm{N}$ did not affect plant height and the production of rhizomes. The yield decreased from $25 \mathrm{t} / \mathrm{ha}$ in the $0.05 \mathrm{~m}$ within-row spacing to $18 \mathrm{t} / \mathrm{ha}$ in $0.40 \mathrm{~m}$ spacing. Productivity increase obtained in within-row spacing smaller than $0.10 \mathrm{~m}$ did not overcome the higher expenses with rhizomes for the respective plantings.
\end{abstract}

Keywords: Curcuma longa, turmeric, plant population, mineral fertilizer, medicinal plants.

\section{(Recebido para publicação em 30 de outubro de 2002 e aceito em 10 de outubro de 2003)}

\begin{abstract}
A crescente utilização da cúrcuma (Curcuma longa L.) na indústria de alimentos tem despertado interesse na expansão dessa cultura e obtenção de produto de melhor qualidade e mais competitivo no mercado. No município de Mara Rosa (GO), essa cultura tem sido conduzida por pequenos produtores e, normalmente, empregando mão-de-obra familiar, constituindo im-
\end{abstract}

portante fonte de renda para a população. Seu cultivo tem sido feito de forma empírica, somente com experiências de produtores. Na literatura poucos trabalhos têm sido desenvolvidos, não somente nas condições locais, como também no Brasil.

Com relação ao espaçamento de plantio, Carvalho et al. (2001), em Lavras (MG), estudando distâncias entre linhas e entre plantas, encontraram maior produtividade de rizomas no espaçamento de $0,20 \mathrm{~m}$ entre plantas, mas não houve influência do espaçamento entre linhas. Cecílio Filho (1996), estudando épocas de plantio e espaçamentos entre plantas, encontrou interação entre estes fatores, com curva quadrática de resposta de produção de rizomas por área nos plantios de outubro

1 Trabalho financiado pelo CNPq. 
e novembro, e curva linear decrescente nos plantios de dezembro e janeiro.

Outros fatores que têm contribuído para o aumento de produtividade são os níveis de adubação química e/ou orgânica. Segundo Peter (1998), na Índia as recomendações de adubação ficam em torno de 280; 155 e $325 \mathrm{~kg} / \mathrm{ha}$ de N, P e $\mathrm{K}$, respectivamente. Estas quantidades incluem nutrientes da adubação orgânica e mineral e resultam em produtividade média de 10,7 t/ha; já Sadanadan (1994) aponta como ótimas as aplicações de 60; 30 e 90 kg/ha de N, P e K, respectivamente, para essa cultura. Rama-Rao e Reddy (1977), utilizando a cultivar CL 326, obtiveram respostas de produção para $\mathrm{N}$ até $375 \mathrm{~kg} / \mathrm{ha}$, para $\mathrm{P}$ até $175 \mathrm{~kg} / \mathrm{ha}$ de $\mathrm{P}_{2} \mathrm{O}_{5}$ e para $\mathrm{K}$ até $237,5 \mathrm{~kg} / \mathrm{ha}$ de $\mathrm{K}_{2} \mathrm{O}$, com produtividade média variando de 24,73 a 39,88 t/ha de rizomas frescos. Porém, Saha (1988), utilizando a cutivar CL 328, comparando ausência de fertilizantes, adubação orgânica com 50 t/ha de esterco bovino, adubação mineral com $90 \mathrm{~kg} / \mathrm{ha}$ de $\mathrm{N}$, $60 \mathrm{~kg} / \mathrm{ha}$ de $\mathrm{P}_{2} \mathrm{O}_{5}$ e $90 \mathrm{~kg} / \mathrm{ha}$ de $\mathrm{K}_{2} \mathrm{O}$ e associação da adubação orgânica com mineral, obteve resposta de produção com produtividade média variando de 4,67 t/ha, sem fertilizantes, a 11,73 t/ha com adubação orgânica isolada.

No Brasil, Goto (1993), estudando épocas de plantio e adubação, relata que em solo com $42 \mathrm{mg} / \mathrm{cm}^{3}$ de $\mathrm{P}$, a resposta de produção de rizomas pelo efeito de adubação fosfatada foi baixa, inferindo que em solos com teores semelhantes, a cultura responde pouco ao $\mathrm{P}$ aplicado ao solo. Goto e Castellane (1996), estudando adubação nitrogenada, obtiveram resposta positiva com $30 \mathrm{~kg} / \mathrm{ha}$ de $\mathrm{N}$, mas não com 60 kg/ha.

Silva et al. (2001), cultivando cúrcuma em Latossolo Vermelho Amarelo de fertilidade média, relataram que a irrigação aumentou a produção de rizomas e pó de cúrcuma, mas as doses de adubo mineral NPK no plantio (até 1.000 kg/ha de 4-14-8) não influíram no crescimento das plantas e na produção de cúrcuma.

Os solos mais utilizados para o cultivo de cúrcuma em Mara Rosa são de boa fertilidade natural, chamados de "terra de cultura". Como os trabalhos citados anteriormente foram realizados em condições de clima e solo diferentes das ocorrentes nesta região, surgiu a necessidade de se estudar os fatores adubação e população de plantas de cúrcuma nas condições edafoclimáticas do estado de Goiás, de modo a contribuir para o aprimoramento das recomendações técnicas para a cultura de cúrcuma.

O objetivo deste trabalho foi avaliar o crescimento e a produção da cúrcuma, em função da adubação nitrogenada, fosfatada e potássica, da densidade de plantio e do parcelamento da adubação nitrogenada.

\section{MATERIAL E MÉTODOS}

Foram conduzidos dois experimentos em condições de campo, na Universidade Federal de Goiás, em Goiânia. O primeiro realizado de janeiro a agosto/ 98 , estudando os fatores (a) doses de $\mathrm{N}$ $(0 ; 40 ; 80 ; 120 ; 160$ e $200 \mathrm{~kg} / \mathrm{ha}) ;(b)$ doses de $\mathrm{P}_{2} \mathrm{O}_{5}(0 ; 50 ; 100 ; 150 ; 250$ e $400 \mathrm{~kg} / \mathrm{ha})$; (c) doses de $\mathrm{K}_{2} \mathrm{O}(0 ; 40 ; 80$; $120 ; 200 ; 280 \mathrm{~kg} / \mathrm{ha}) ;(\mathrm{d})$ densidades de plantio $(55.556$; 41.667 e 33.333 plantas/ha, no espaçamento de $0,60 \mathrm{~m}$ entre linhas e 71.428 ; 47.619 e 35.714 plantas/ha no espaçamento de $0,70 \mathrm{~m}$ entre linhas) e (e) parcelamento da adubação nitrogenada [toda no plantio; $1 / 2$ no plantio e $1 / 2$ aos três meses após o plantio (AP); $1 / 3$ no plantio, $1 / 3$ aos dois meses e $1 / 3$ aos quatro meses AP; $1 / 4$ no plantio, $1 / 4$ a um mês e meio, $1 / 4$ aos 3 meses e $1 / 4$ aos quatro meses e meio AP.

Os tratamentos, em número de 24, foram arranjados em uma matriz experimental do tipo Baconiana, como relatado por Vieira e Lima (1987) e dispostos no delineamento de blocos casualizados com quatro repetições. A parcela experimental foi constituída de quatro linhas de plantas com quatro metros de comprimento, sendo consideradas úteis as duas linhas centrais excluindo-se $0,5 \mathrm{~m}$ das extremidades de cada linha.

As respostas às doses de $\mathrm{N}, \mathrm{P}$ e $\mathrm{K}$ foram analisadas por meio de regressão, enquanto as respostas aos tratamentos de densidades de plantio e épocas de adubação através da comparação de médias pelo teste de Tukey. Foram uti- lizados uréia, superfosfato triplo e cloreto de potássio como fontes de nitrogênio, fósforo e potássio, respectivamente. Foram aplicados $50 \mathrm{~kg} / \mathrm{ha}$ de FTE BR-12, em toda a área experimental. De acordo com os tratamentos, a adubação de plantio foi distribuída e incorporada no sulco imediatamente antes do plantio e a de cobertura, de acordo com o parcelamento de N, foram feitas aplicando-se as respectivas quantidades do adubo entre as linhas de plantas.

Os resultados das análises de fertilidade, de amostras do solo do primeiro experimento coletadas na profundidade de 0 a $20 \mathrm{~cm}$, no local experimental, são: 4,2\% de M.O.; 6,5\% de pH em água; $21,3 \mathrm{mg} / \mathrm{dm}^{3}$ de $\mathrm{P}$ e teores de 4,$0 ; 4,1$; 2,0; 2,4 e 12,5 $\mathrm{cmol} / \mathrm{dm}^{3}$ de $\mathrm{K}, \mathrm{Ca}, \mathrm{Mg}$; $\mathrm{H}+\mathrm{Al}$ e CTC, respectivamente. A análise física mostrou teores de 61,10 e $29 \%$ de areia, silte e argila, respectivamente, indicando tratar-se de solo franco argilo arenoso.

O segundo experimento foi conduzido de dezembro/99 a setembro/00, estudando-se dois espaçamentos entre linhas $(0,60$ e $0,80 \mathrm{~m})$ e seis espaçamentos entre plantas na linha $(0,05 ; 0,10 ; 0,15 ; 0,20 ; 0,30$ e $0,40 \mathrm{~m})$ arranjados em fatorial de 2 x 4 com quatro repetições, no delineamento de blocos casualizados, com parcelas de quatro linhas de plantas com quatro metros de comprimento. Foram consideradas úteis as plantas dos três metros centrais das duas linhas internas.

O plantio foi feito na profundidade média de 5,0 cm e utilizando-se rizomas secundários, com peso médio de $13 \mathrm{~g}$ no primeiro experimento e $15 \mathrm{~g}$ no segundo.

Foram estudados o gasto de rizomas para plantio, altura de plantas aos 100 dias no primeiro experimento e aos 60 e aos 100 dias no segundo experimento e produção de rizomas frescos após a colheita e produção de rizomas secos após secagem em estufa a $60^{\circ} \mathrm{C}$ até peso constante, nos dois experimentos.

\section{RESULTADOS E DISCUSSÃO}

No primeiro experimento, a altura das plantas foi influenciada apenas pelas doses de $\mathrm{N}$, ocorrendo aumento de 
acordo com as doses segundo a equação $\bar{Y}=64,63+0,0319 X$ com $\mathrm{R}^{2}=0,71$, sendo a maior altura $(71 \mathrm{~cm})$, obtida com a dose de $200 \mathrm{~kg} / \mathrm{ha}$ de N. Segundo Marschner (1995), esse crescimento da parte aérea pode retardar e até reduzir o armazenamento de fotoassimilados em raízes, tubérculos e rizomas.

As doses de $\mathrm{N}$ aumentaram, num efeito quadrático, a produção de rizomas frescos, até atingir o máximo estimado de $16.124 \mathrm{~kg} / \mathrm{ha}$ com a dose média de $130 \mathrm{~kg} / \mathrm{ha}$, correspondendo a 33\% de aumento na produção em relação à sua ausência. Porém, esse efeito não foi observado na produção de rizomas secos que é a forma comercializada (Figura 1). A maior produção de rizomas frescos, em função do $\mathrm{N}$ foi, em parte, devido ao teor de umidade dos rizomas estimado pela equação $\bar{Y}=77,87+0,0086 X$ com $\mathrm{R}^{2}=0,88$, que cresceu de $77,87 \%$ na dose zero de $\mathrm{N}$ até $79,59 \%$ na dose de $200 \mathrm{~kg} / \mathrm{ha}$. Apesar do crescimento da parte aérea em altura e dos rizomas em peso fresco, devido ao $\mathrm{N}$, não se constatou diferenças de peso seco nos rizomas. Esse crescimento dos rizomas em peso fresco foi em função do maior teor de umidade. Marschner (1995) afirma que a adubação nitrogenada em algumas culturas rizomatosas nem sempre resulta em maior crescimento de matéria seca no rizoma. Em algumas culturas tuberosas, a produção de rizomas e raízes nem sempre acompanha o crescimento vegetativo devido a adubações nitrogenadas (Raij, 1991).

A produção de rizomas frescos, em função das doses de P, foi melhor ajustada ao modelo raiz quadrático, crescendo de $12.799 \mathrm{~kg} / \mathrm{ha}$ na dose zero, até atingir o ponto máximo de $15.763 \mathrm{~kg} /$ ha na dose de $177 \mathrm{~kg} / \mathrm{ha}$, correspondendo a um aumento de 23\% (Figura 2). O solo utilizado nesse ensaio apresentava teores médios de $\mathrm{P}$ contribuindo para esse aumento na produção. De modo semelhante ao nitrogênio, o fósforo não influenciou a produção de rizomas secos, porém esse mesmo nutriente não influenciou significativamente o teor de umidade dos rizomas colhidos. Goto (1993), também encontrou pequeno efeito de adubação fosfatada no desenvolvimento de cúrcuma, sugerindo que a cultura seja pouco exigente em fósforo.

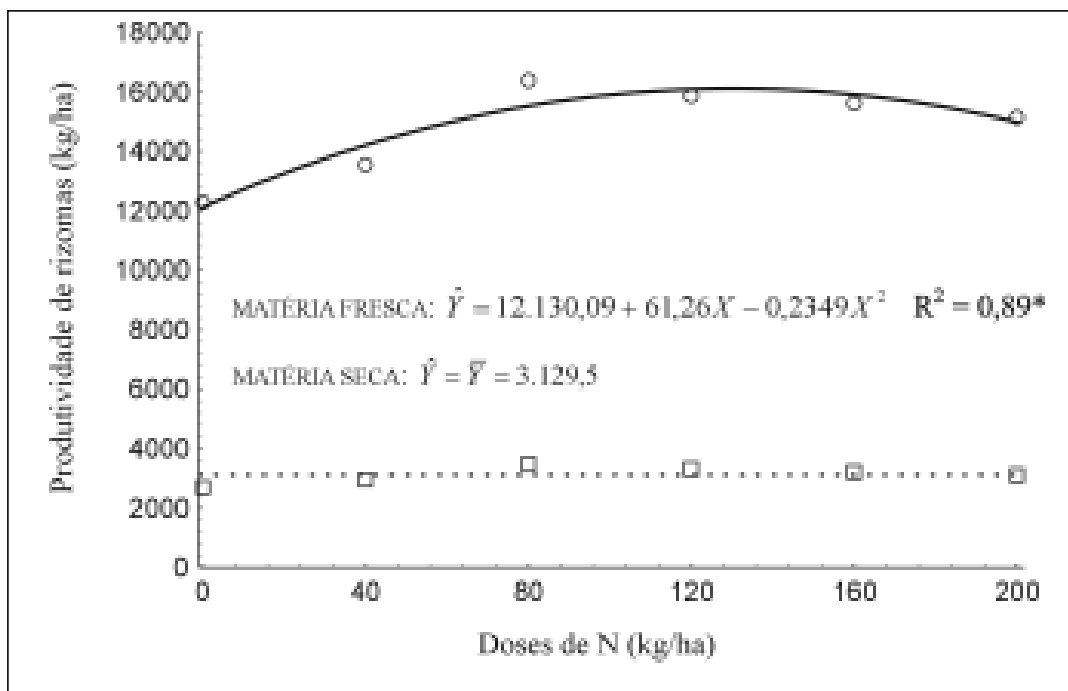

Figura 1. Produtividade de matéria fresca e seca de rizomas de cúrcuma (Curcuma longa L.), em função de doses de N. Goiânia, UFG, 1998.

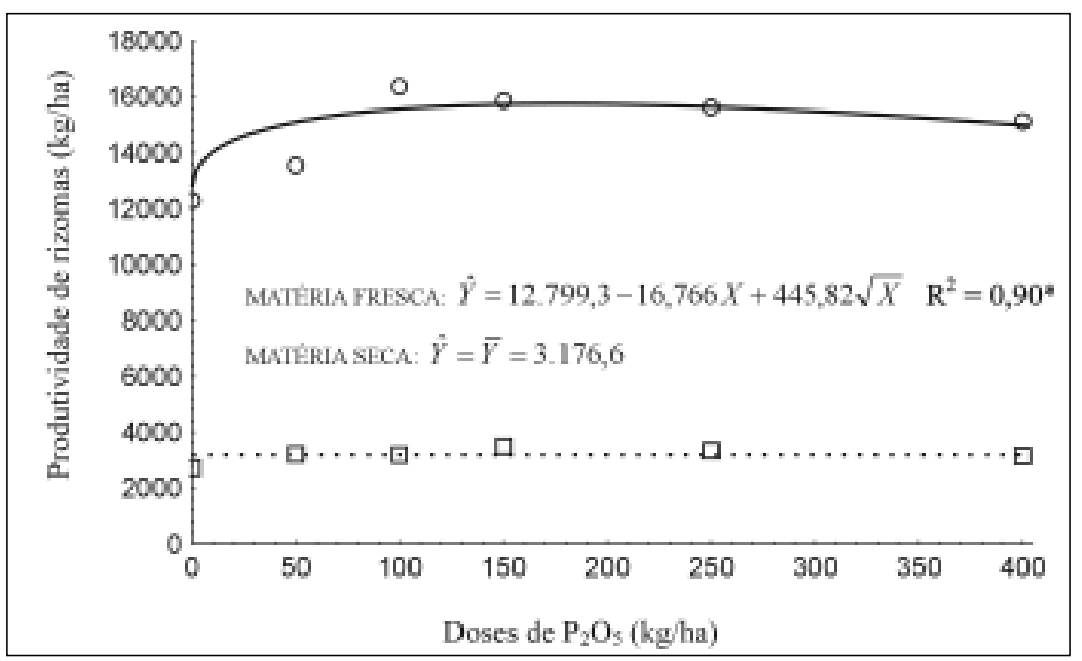

Figura 2. Produtividade de matéria fresca e seca de rizomas de cúrcuma (Curcuma longa L.), em função de doses de $\mathrm{P}_{2} \mathrm{O}_{5}$. Goiânia, UFG, 1998.

Não foram observados efeitos significativos das doses de $\mathrm{K}$ e do parcelamento de $\mathrm{N}$ nas características avaliadas. O solo utilizado nesse ensaio apresentava teores altos de $\mathrm{K}$, o que contribuiu para a ausência de efeito significativo das doses de $\mathrm{K}$ nas características avaliadas. Nas condições da Índia, com baixos teores de K no solo, como relatado por Sadanadan (1994) e Peter (1998), esse elemento parece ser um dos mais importantes na adubação dessa cultura.

De modo geral, a cultura de cúrcuma tem mostrado fracas respostas de produção de rizomas à aplicação de fertili- zantes minerais (Goto, 1993; Silva et al., 2001); uma das possíveis causas pode ser o fato de que a cultura, sendo propagada por via vegetativa, tenha acumulado doenças viróticas e outras sem sintomas claros, que limitam o potencial produtivo, à semelhança do que ocorre com a cultura de batata (Câmara e Filgueira, 1986). A cultura de ápices caulinares "in vitro" pode originar material propagativo com melhor qualidade fitossanitária que apresente melhores respostas à adubação.

No segundo experimento o efeito sobre a altura das plantas (Figura 3), foi 


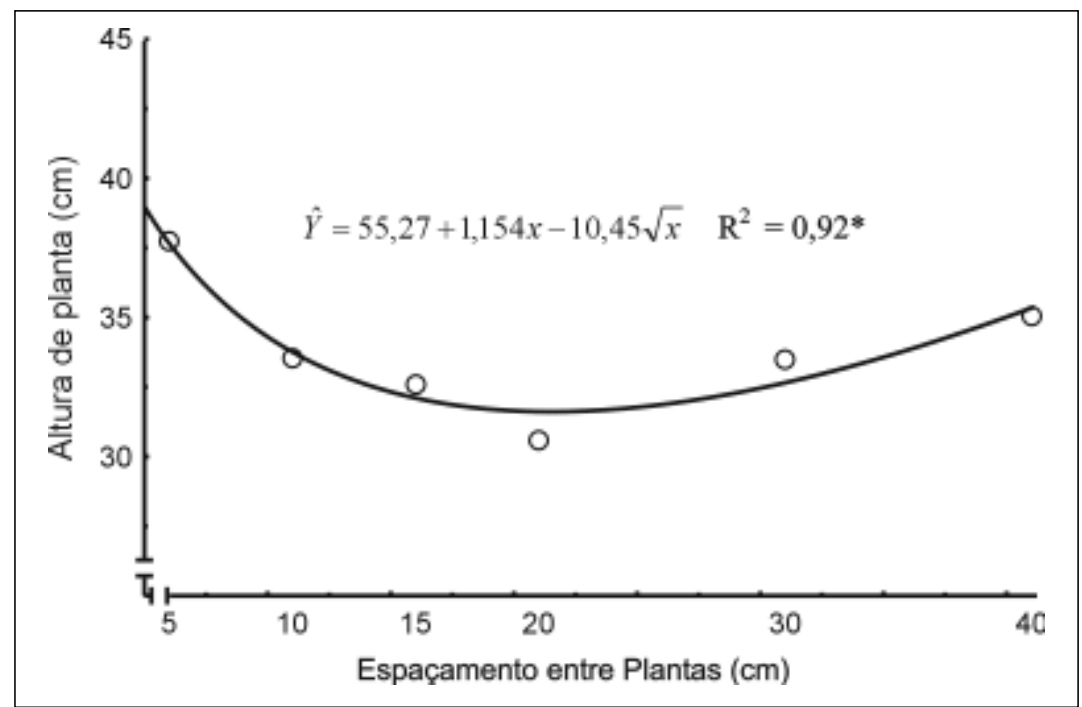

Figura 3. Altura de planta de cúrcuma (Curcuma longa L.), aos 60 dias após o plantio, em função do espaçamento entre plantas na linha. Goiânia, UFG, 2000.

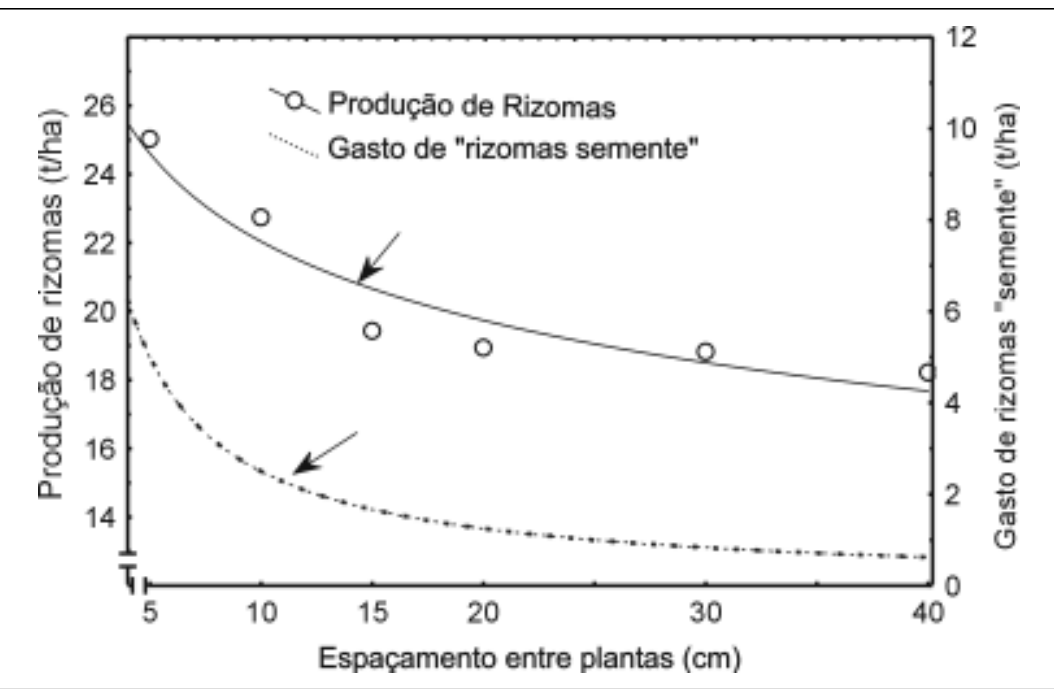

Figura 4. Gasto de rizomas "semente" no plantio e produção de rizomas na colheita de cúrcuma (Curcuma longa L.), em função do espaçamento entre plantas na linha. Goiânia, UFG, 2000.

melhor explicado pelo modelo raiz quadrático, que apontou decréscimo, partindo de $38 \mathrm{~cm}$ no espaçamento de $0,05 \mathrm{~m}$ para o ponto mínimo de $32 \mathrm{~cm}$ no espaçamento de $0,20 \mathrm{~m}$, provavelmente devido ao efeito de concorrência pela luz entre as plantas, também decrescente com o aumento do espaçamento. A partir do espaçamento de $0,20 \mathrm{~m}$ até $0,40 \mathrm{~m}$, as plantas mostraram tendência de crescimento em altura em função da maior disponibilidade de espaço para as plantas, nesse caso talvez devido à maior disponibilidade de luz e de outros fatores. Nesse experimento, a altura das plantas, avaliada aos 100 dias, não foi influenciada pelas densidades de plantio. É possível que o perfilhamento das plantas, não avaliado nesses experimentos, que normalmente ocorre entre 60 a 100 dias após o plantio, seja um fator que compense em parte espaçamentos de plantio maiores.

No segundo experimento, a produtividade de rizomas frescos foi significativamente influenciada apenas pelo fator espaçamento entre plantas, decrescendo com o aumento dos espaçamentos de plantio, segundo a função potencial apresentada na Figura 4. Os dados mostraram uma tendência de estabilização da produtividade de rizomas entre os espaçamentos de 0,15 a $0,40 \mathrm{~m}$. É possível que nessa faixa de espaçamento o perfilhamento das plantas tenha compensado os espaçamentos maiores. Cecílio Filho (1996), estudando diferentes densidades de plantio de cúrcuma, observou que a produção de rizomas dependeu da época de plantio e que o peso de matéria seca do rizoma, nos plantios realizados em dezembro e janeiro, não variou com as densidades de plantio de $62.500 ; 35.714$ e 25.000 plantas ha ${ }^{-1}$, concordando com os resultados encontrados neste trabalho.

Na Figura 4 também é apresentada a estimativa de gasto de rizomas em função dos espaçamentos de plantio. Verificou-se que o ganho de produção pela redução do espaçamento de $0,10 \mathrm{~m}$ para $0,05 \mathrm{~m}$ na linha de plantio não superou, significativamente, os gastos de rizomas no plantio. Considerando ainda o transporte e a mão-de-obra para plantar maior quantidade de rizomas em espaçamentos menores, os ganhos de produção podem, às vezes, não ser compensadores. Assim, parecem mais adequados espaçamentos de plantio entre plantas na linha não menores que 10 centímetros, que resultam em gastos de rizomas (peso médio de $15 \mathrm{~g}$ ) em torno de 2,5 t/ ha. Govinden e Cheong (1995), estudando tipos e quantidade de rizomas para o plantio de cúrcuma, encontraram, como ótimo, $3 \mathrm{t} / \mathrm{ha}$, independente do tipo de rizoma.

Nas condições dos experimentos relatados, concluiu-se que o nitrogênio aumenta até $33 \%$ e o fósforo em até $23 \%$, a produtividade de rizomas fres$\cos$, mas não influenciaram a produtividade de rizomas secos. O potássio e o parcelamento de $\mathrm{N}$ não influenciam, significativamente, a altura das plantas e a produção de rizomas. Espaçamentos de plantio menores que $0,15 \mathrm{~m}$ entre plantas resultam em maior produtividade, mas os ganhos de produtividade não compensam os gastos com rizomas no plantio, em espaçamentos menores que $0,10 \mathrm{~m}$ entre plantas. Os espaçamentos 
entre linhas estudados não influenciam significativamente a produção de rizomas.

\section{LITERATURA CITADA}

CÂMARA, F.A.; FILGUEIRA, F.A.R. Comportamento de cultivares européias de batata em gerações sucessivas. Horticultura Brasileira, Brasília, v.4, n.1, p.7-9, 1986

CARVALHO, C.M.; SOUZA, R.J.; CECÍLIO FILHO, A.B. Produtividade da cúrcuma (Curcuma longa $\mathrm{L}$.) cultivada em diferentes densidades de plantio. Ciência e Agrotecnologia, n.25, v.2, p.330335, 2001.

CECÍlIO FILHO, A.B. Época e densidade de plantio sobre a fenologia e o rendimento do cúrcuma (Curcuma longa L.). Lavras: Faculdade de Engenharia Agrícola. 1996. 100 p. (Tese doutorado)

GOTO, R. Épocas de plantio, adubação fosfatada e unidades térmicas em cultura de açafrão (Curcuma longa L.). Jaboticabal: Faculdade de Ciências Agrárias e Veterinárias, 1993.93 p. (Tese doutorado).
GOTO, R.; CASTELLANE, P.D. Sistema de cultivo e adubação nitrogenada em cobertura na cultura de açafrão (Curcuma longa $\mathrm{L}$.). In: CONGRESSO LATINO AMERICANO DE RAIZES TROPICAIS, 1., CONGRESSO BRASILEIRO DE MANDIOCA, 9., 1996, São Pedro, SP. Anais... 1996. p. 156.

GOVINDEN, N.; CHEONG, K.W.Y. Matériel de plantation et taux optimum de semences du curcuma. Revue Agricole et Sucriere de l'Ile Maurice. v.74, n.3, p.1-8,1995.

MARSCHNER, H. Mineral nutrition of higher plants. 2. ed. London: Academic Press, 1995. 889

PETER, K.V. Spices research in India. Indian Journal of Agricultural Sciences, v.68, n.8, p.527532, 1998.

RAMA-RAO, M.; REDDY, V.R. Effect of different levels of nitrogen, phosphorus, and potassium on yield of turmeric (Curcuma longa L.). Journal of Plantation Crops, v.5, n.1, p.6062, 1977.
SADANADAN, A.K. Doubling our production of spices aiming through infrastructure spread and research: AICRP Spices. Indian Horticulture, v.39, n.3, p.10-16, 1994

SAHA, A.K. Note on response of turmeric to manure and source of $\mathrm{N}$ and $\mathrm{P}$ under terrace conditions of mid-altitude Mizoram. Indian Journal of Horticulture, v.45, n.1 e 2, p.139-140, 1988.

SILVA, N.F.; NASCIMENTO, J.L.; ROLIM, H.M.V.; SONNENBERG, P.E.; BORGES, J.D Produção de cúrcuma em função de irrigação e adubação mineral. Horticultura Brasileira, Brasília, v.19, n.2, p.233, 2001.

RAIJ, B. Fertilidade do solo e adubação. São Paulo: Agronômica Ceres, Potafos, 1991. 343 p.

VIEIRA, R.F.; LIMA, P.C. Uso da estatística na avaliação da fertilidade do solo. Viçosa: UFV. 1987. 104 p. 